\title{
Tracking Control for a Piezoelectric Nanopositioner Using Estimated States and Feedforward Compensation of Hysteresis *
}

\author{
Arnfinn Aas Eielsen* Jan Tommy Gravdahl* \\ Kristin Y. Pettersen* Lukas Vogl ${ }^{*}$ \\ * Department of Engineering Cybernetics, Norwegian University of \\ Science and Technology, 7491 Trondheim, Norway \\ (e-mail: \{eielsen,tommy.gravdahl,kristin.y.pettersen\}@itk.ntnu.no, \\ vogl@stud.ntnu.no).
}

\begin{abstract}
Nanopositioning stages utilizing piezoelectric actuators exhibit several undesired features inhibiting good reference tracking performance. The most salient features are lightly damped mechanical resonances, hysteresis, and creep. In addition, sensor noise can limit the resolution achievable when applying closed-loop control schemes. In order to reduce sensor noise when using closed-loop control, we develop a state estimator in the form of an adaptive Luenberger observer. Furthermore, we propose a novel method for compensating the hysteretic behavior in piezoelectric actuators when tracking a reference trajectory, and present a method for online identification of the parameters of the system, aiming for simplicity and ease of implementation. The backstepping framework is used to obtain an adaptive control law and to analyze stability and boundedness of the tracking error. Experimental results are presented in order to assess the performance of the proposed hysteresis compensation, as well as the backstepping control law, on a flexure-based nanopositioner using piezoelectric actuators.
\end{abstract}

Keywords: Piezoeletric actuators, nanopositioning, tracking, hysteresis, observers, adaptive control, identification, nonlinear systems.

\section{INTRODUCTION}

Positioning with precision on the nano scale is needed in several applications, such as scanning probe microscopy, nano lithography, and alignment systems. Piezoelectric actuators see widespread use in positioning devices for such applications. This is due to their compact size, high bandwidth, their ability to produce large forces, and their effectively infinite resolution. On the other hand, nanopositioning stages utilizing piezoelectric actuators exhibit several undesired features inhibiting good reference tracking performance. The most salient features are lightly damped mechanical resonances, hysteresis, and creep. In addition, sensor noise limits the resolution achievable when applying closed-loop control schemes (Devasia et al. [2007]).

In this paper we will develop a controller for tracking periodic trajectories with bounded, piecewise continuous derivatives. Triangle wave reference signals are typically used when generating images in scanning probe microscopy. We will focus on reducing hysteresis, and we develop a novel method for feed forward compensation for hysteresis. We address the problem of sensor noise by using a state observer, and in order to increase precision we employ an adaptive controller.

We start by developing models that capture the hysteretic behavior and the mechanical dynamics. To suppress sensor noise, we set up an adaptive Luenberger observer (Ioannou and Sun [1995]) to compute state estimates, and we tune it by using the steady-state Kalman filter (Simon [2006]) with a nominal model. Considering the hysteretic

* This work was supported by the Norwegian Research Council and the Norwegian University of Science and Technology. behavior as a bounded disturbance, an ISS (input-to-statestability) backstepping controller (Krstic et al. [1995]) is utilized to obtain tracking. This controller is shown to be robust against parameter uncertainty and bounded disturbances. It provides bounds for the tracking error and it allows for modularized parameter identification. Parameter identification using normalized least-squares is then employed in order to improve both state estimates and tracking performance. Lastly we propose a simple method to compensate for the disturbance due to the hysteric behavior, and thus reduce the tracking error.

A diagram of the proposed control structure is presented in Fig. 1.

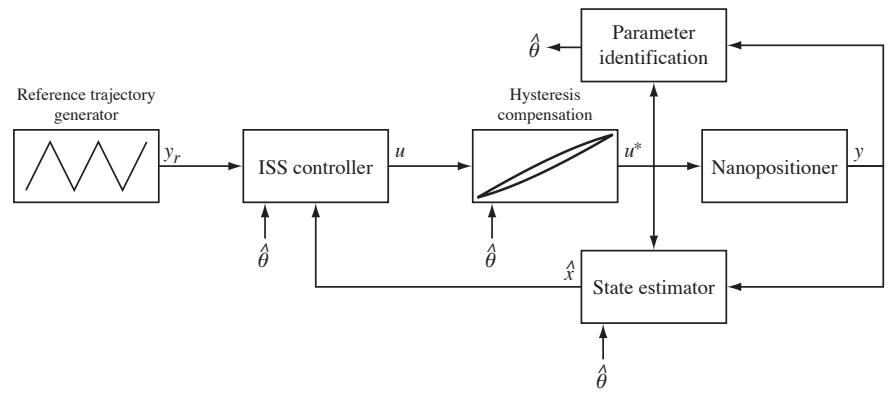

Fig. 1. The proposed control structure.

\section{MODELING}

In this section we present a model for the system at hand, which is a flexure based nanopositioning stage with a piezoelectric stack actuator. The dynamics of a 
given point $x$ on the mechanical system attached to the actuator can adequately be described as a linear lumped parameter system with an infinite number of resonance frequencies. We will use reference signals with a fundamental frequency below the first resonance frequency of the given nanopositioning stage. Mechanical resonances above the first resonance of the stage will therefore be considered negligible, and a second order system should provide a good approximation.

The hysteretic behavior of piezoelectric actuators is due to ferroelectric phenomena, and therefore the hysteresis exhibited in such actuators will appear between applied voltage and induced charge (Newcomb and Flinn [1982]). The mechanical response, the force developed by the actuator, considering voltage as input, will therefore exhibit hysteresis, but the response considering charge as input, is linear. To account for the hysteretic behavior between applied voltage and induced charge, we use a modified version of the Coleman-Hodgdon equations, which has been shown to be a good phenomenological model in this context (Banning et al. [2001]). The modified version of the Coleman-Hodgdon equations is shown to be equivalent to the original formulation, but is on a form that makes it possible to use it for feed forward hysteresis compensation.

\subsection{Piezoelectric Stack Actuator}

The response of a piezoelectric stack actuator, can be described by its constitutive equations (Preumont [2006])

$$
\begin{aligned}
\varepsilon & =s^{E} \sigma+d_{33} E \\
D & =d_{33} \sigma+\kappa^{\sigma} E,
\end{aligned}
$$

where $s^{E}$ is the elastic compliance $\left[\mathrm{m}^{2} / \mathrm{N}\right], d_{33}$ is a piezoelectric constant $[\mathrm{m} / \mathrm{V}]$, and $\kappa^{\sigma}$ is the permittivity of the material $[\mathrm{F} / \mathrm{m}]$. We assume that the strain $\varepsilon[\mathrm{m} / \mathrm{m}]$, stress $\sigma\left[\mathrm{N} / \mathrm{m}^{2}\right]$, electric field $E[\mathrm{~V} / \mathrm{m}]$, and charge density $D$ $\left[\mathrm{C} / \mathrm{m}^{2}\right]$ are applied or measured along the poling axis of the piezoelectric material.

Blocking Force The blocking force of the piezoelectric stack actuator is the maximum force that can be developed by the actuator in response to an applied voltage, and can be found when the actuator is clamped, $\varepsilon=0$.

A piezoelectric stack actuator consists of multiple layers of piezoelectric material. Considering one element, or layer, of the stack, we find the stress on that element due to an applied electric field from (1) as

$$
\sigma=-d_{33} c^{E} E
$$

where $c_{E}=\frac{1}{s^{E}}$ is the elastic stiffness $\left[\mathrm{N} / \mathrm{m}^{2}\right]$.

The geometry of one stack element is a rectangular cuboid, with thickness $l[\mathrm{~m}]$ along the poling direction, and surface area $A\left[\mathrm{~m}^{2}\right]$ for the faces normal to the poling direction. The stress on one element due to a force $F_{a}[\mathrm{~N}]$ in the poling direction is therefore $\sigma=\frac{F_{a}}{A}$. The electric field due to the an applied voltage $u[\mathrm{~V}]$ is $E=\frac{u}{l}$. For one stack element in a balance of stress, we have

$$
\frac{F_{a}}{A}-d_{33} c^{E} \frac{u}{l}=0
$$

and for a stack with $n$ elements with a total thickness of $\ell=n l$ the blocking force will be

$$
F_{a}=n d_{33} k_{a} u=K_{a} u, \quad k_{a}=\frac{c^{E} A}{\ell},
$$

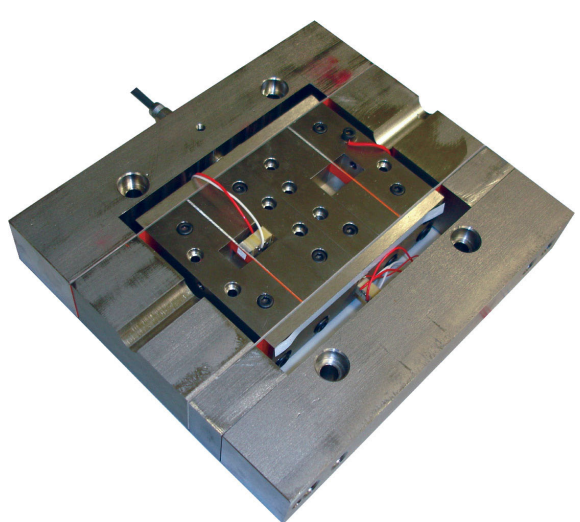

Fig. 2. Nanopositioner.

where we recognize $k_{a}$ as the stiffness $[\mathrm{N} / \mathrm{m}]$ of the stack, and we lump the various material parameters into the gain coefficient $K_{a}=n d_{33} k_{a}$ for convenience.

Charge The charge density on the electrodes of an element in the piezoelectric stack actuator is given by (2). For the stack with $n$ elements, the total charge $q=n A D$ [C], given the geometry above, will be

$$
q=n d_{33} F+\frac{n^{2} \kappa^{\sigma} A}{\ell} u .
$$

The stress in the material due to strain is given by Hooke's law as

$$
\sigma=c^{E} \varepsilon
$$

and for the given geometry the resulting force due to a displacement in the stack $x_{1}[\mathrm{~m}]$ must be

$$
F_{a}=\frac{1}{n} \frac{c^{E} A}{l} x=k_{a} x_{1},
$$

thus the total charge in the stack is

$$
q=n d_{33} k_{a} x+C_{a} u=K_{a} x_{1}+C_{a} u,
$$

where $C_{a}=\frac{n^{2} \kappa^{\sigma} A}{\ell}$ is the capacitance $[\mathrm{F}]$ of the stack.

\subsection{Nanopositioning Stage}

If we assume that the combined mass of the moving platform and the piezoelectric stack actuator is $M=m_{p}+$ $m_{a}[\mathrm{~kg}]$, the combined damping coefficient of the flexures and the actuator is $c=c_{f}+c_{a}[\mathrm{Ns} / \mathrm{m}]$, and the combined spring constant of the flexures and the piezoelectric stack is $k=k_{f}+k_{a}[\mathrm{~N} / \mathrm{m}]$, the dynamics of the system is

$$
\begin{aligned}
& \dot{x}_{1}=x_{2} \\
& \dot{x}_{2}=-\frac{k}{M} x_{1}-\frac{c}{M} x_{2}+\frac{1}{M} F_{a}, \quad F_{a}=K_{a} u,
\end{aligned}
$$

or more briefly

$$
\begin{aligned}
& \dot{x}_{1}=x_{2} \\
& \dot{x}_{2}=-a_{0} x_{1}-a_{1} x_{2}+b_{0} u,
\end{aligned}
$$

where $a_{0}=\frac{k}{M} a_{1}=\frac{c}{M}, b_{0}=\frac{K_{a}}{M}$.

If we are considering charge as the input to the above system, we substitute (4) into (5) and obtain

$$
\begin{aligned}
& \dot{x}_{1}=x_{2} \\
& \dot{x}_{2}=-\bar{a}_{0} x_{1}-a_{1} x_{2}+\bar{b}_{0} q
\end{aligned}
$$

with

$$
\bar{a}_{0}=a_{0}+b_{0} \frac{K_{a}}{C_{a}} \text { and } \bar{b}_{0}=b_{0} \frac{1}{C_{a}} .
$$




\subsection{Hysteresis}

The Coleman-Hodgdon equations (Coleman and Hodgdon [1986]) is a well known Duhem model for describing hysteretic behavior in ferromagnetic materials, which also has seen widespread use describing ferroelectric hysteresis as well (e.g. Banning et al. [2001], Stepanenko and Su [1998]).

A formulation of the Coleman-Hodges equations that capture the behavior observed in the system at hand (since we apply voltages below the coercive voltage for the actuator), is

$$
\dot{w}=\beta \dot{u}-\alpha w|\dot{u}|+\gamma|\dot{u}| u
$$

where $u$ is the input, $w$ is the output, and the parameters satisfy $\alpha>0, \beta>0, \frac{\gamma}{\alpha}>\beta$, and $\frac{\gamma}{\alpha} \leq 2 \beta$. The last inequality can be relaxed somewhat, to

$$
\frac{\gamma}{\alpha}-\beta<\beta\left(1-\mathrm{e}^{-2 \alpha U^{*}}\right)^{-1}
$$

where $U^{*}$ in this context can be understood to be the coercive voltage (proportional to the electric field strength that causes change in remanent polarization for a given material) for the piezoelectric stack actuator. This ensures a physical behavior, such that the slope of $\dot{w}$ has the same sign as the slope of $\dot{u}$, that is $\frac{\mathrm{d} w}{\mathrm{~d} u}>0$.

Eq. (8) can be solved explicitly if we observe that it can be written as

$$
\dot{w}=(\beta-\alpha w+\gamma u)(\dot{u})^{+}-(\beta+\alpha w-\gamma u)(\dot{u})^{-}
$$

where $(\dot{u})^{+}=\dot{u}$ and $(\dot{u})^{-}=0$ when $\dot{u} \geq 0$, and $(\dot{u})^{+}=0$ and $(\dot{u})^{-}=\dot{u}$ when $\dot{u}<0$. The dependence on time can then be cancelled and we are left with two linear differential equations for the two cases. For the case $\dot{u} \geq 0$ we find the solution of as

$$
\mathrm{d} w=(\beta-\alpha w+\gamma u) \mathrm{d} u \Rightarrow \frac{\mathrm{d} w}{\mathrm{~d} u}+\alpha w=\beta+\gamma u
$$

$$
\begin{aligned}
w & =\mathrm{e}^{-h}\left[\int \mathrm{e}^{h}(\beta+\gamma u) \mathrm{d} u+C_{1}\right] \\
& =\mathrm{e}^{-\alpha u}\left[\frac{(\alpha \beta-\gamma+\alpha \gamma u) \mathrm{e}^{\alpha u}}{\alpha^{2}}+C_{1}\right],
\end{aligned}
$$

where $h=\int \alpha \mathrm{d} u=\alpha u$ has been used, and which yields

$$
\begin{gathered}
w^{+}=\frac{\gamma}{\alpha} u+\frac{\alpha \beta-\gamma}{\alpha^{2}}+C_{1} \mathrm{e}^{-\alpha u}, \\
w_{0}=\frac{\gamma}{\alpha} u_{0}+\frac{\alpha \beta-\gamma}{\alpha^{2}}+C_{1} \mathrm{e}^{-\alpha u_{0}}, \\
C_{1}=\mathrm{e}^{\alpha u_{0}}\left(w_{0}-\frac{\alpha \beta-\gamma}{\alpha^{2}}-\frac{\gamma}{\alpha} u_{0}\right) .
\end{gathered}
$$

Similarly, for the case $\dot{u}<0$ we have the solution

$$
\begin{gathered}
w^{-}=\frac{\gamma}{\alpha} u+\frac{\gamma-\alpha \beta}{\alpha^{2}}+C_{2} \mathrm{e}^{\alpha u}, \\
C_{2}=\mathrm{e}^{-\alpha u_{0}}\left(w_{0}-\frac{\gamma-\alpha \beta}{\alpha^{2}}-\frac{\gamma}{\alpha} u_{0}\right) .
\end{gathered}
$$

From the solutions of the equations we see that the output of (8) can be written

$$
w=\frac{\gamma}{\alpha} u+w_{h}
$$

and we observe that $w_{h}$ can be taken to be the solution of the differential equation

$$
\dot{w}_{h}=-\bar{\beta} \dot{u}-\bar{\alpha} w_{h}|\dot{u}|
$$

where we choose

$$
\bar{\alpha}=\alpha \text { and } \bar{\beta}=\frac{\gamma-\alpha \beta}{\alpha}>0 .
$$

Equation (12) is similar to a case of the well known Dahl solid friction model (Dahl [1968]), except for the sign of the parameter $\bar{\beta}$. This equation can also be solved for the cases $\dot{u} \geq 0$ and $\dot{u}<0$ in a similar fashion as above, using e.g. separation of variables. The solution for $\dot{u} \geq 0$ is

$$
\begin{aligned}
& w_{h}^{+}=\frac{1}{\bar{\alpha}}\left(-\bar{\beta}-C_{3} \mathrm{e}^{-\bar{\alpha} u}\right)=\frac{\alpha \beta-\gamma}{\alpha^{2}}-\frac{1}{\alpha} C_{3} \mathrm{e}^{-\alpha u}, \\
& C_{3}=\mathrm{e}^{\bar{\alpha} u_{0}}\left(-\bar{\beta}-\bar{\alpha} w_{h 0}\right) \\
& =-\alpha \mathrm{e}^{\alpha u_{0}}\left(w_{0}-\frac{\alpha \beta-\gamma}{\alpha^{2}}-\frac{\gamma}{\alpha} u_{0}\right),
\end{aligned}
$$

and for $\dot{u}<0$ it is

$$
\begin{gathered}
w_{h}^{-}=\frac{1}{\bar{\alpha}}\left(\bar{\beta}-C_{4} \mathrm{e}^{\bar{\alpha} u}\right), \\
C_{4}=\mathrm{e}^{-\bar{\alpha} u_{0}}\left(\bar{\beta}-\bar{\alpha} w_{h 0}\right) .
\end{gathered}
$$

We see that the solutions of (11) and (12) are the same as the solutions of (8).

Since we know that the hysteretic behavior in a piezoelectric actuator occurs between applied voltage $u$ and induced charge $q$, we propose to model the charge in such an actuator as

$$
\begin{aligned}
\dot{u} & =v \\
\dot{q}_{h} & =-b v-a q_{h}|v| \\
q & =c u+q_{h},
\end{aligned}
$$

where

$$
a=\alpha, b=\frac{\gamma-\alpha \beta}{\alpha}, \text { and } c=\frac{\gamma}{\alpha} .
$$

By using (15) as the model for the charge in the system, the linearized behavior of (15) should be an approximation of (4), that is

$$
\left(C_{a}+K_{a} G(s)\right) u \approx K_{q} u,
$$

where $G(s)$ is the Laplace transform of (5),

$$
G(s)=\frac{x_{1}}{u}(s)=\frac{b_{0}}{s^{2}+a_{1} s+a_{0}},
$$

and $K_{q}$ is the linearized sensitivity of (15). This should be a good approximation as long as we are exciting the system at frequencies well below the first resonance of the system.

Remark If we choose a storage function such as

$$
V=\frac{1}{2}\left(c u^{2}+\frac{1}{b} q_{h}^{2}\right)+u q_{h},
$$

the total derivative of $V$ along the trajectories of (15) is

$$
\begin{aligned}
\dot{V}=c u v & +\frac{1}{b} q_{h}\left(-b v-a q_{h}|v|\right)+u \dot{q}_{h}+v q_{h} \\
& =\left(c v+\dot{q}_{h}\right) u-\frac{a}{b} q_{h}{ }^{2}|v|=\dot{q} u-\frac{a}{b} q_{h}{ }^{2}|v| \leq \dot{q} u
\end{aligned}
$$

thus, the model (15) is passive from $u$ to $\dot{q}$.

The energy expended in a single traversal of the hysteresis loop (see Fig. 3 ), if the applied voltage $u$ cycle between a some minimum $u\left(\tau_{0}\right)=u\left(\tau_{2}\right)=u_{m}$ and some maximum $u\left(\tau_{1}\right)=u_{M}$ during a period $\tau_{2}-\tau_{0}$, can be found from the work done, which is 


$$
\begin{aligned}
W & =-\int_{\tau_{0}}^{\tau_{2}} q v \mathrm{~d} t=-\int_{\tau_{0}}^{\tau_{1}} q v \mathrm{~d} t-\int_{\tau_{1}}^{\tau_{2}} q v \mathrm{~d} t \\
& =-\int_{u_{m}}^{u_{M}} q^{+} \mathrm{d} u-\int_{u_{M}}^{u_{m}} q^{-} \mathrm{d} u \\
& =-\underbrace{\int_{u_{m}}^{u_{m}} c u \mathrm{~d} u}_{=0}-\int_{u_{m}}^{u_{M}} q_{h}^{+} \mathrm{d} u-\int_{u_{M}}^{u_{m}} q_{h}^{-} \mathrm{d} u .
\end{aligned}
$$

Since $q_{h}^{+}\left(u_{M}\right)=q_{h}^{-}\left(u_{M}\right)$ and $q_{h}^{+}\left(u_{m}\right)=q_{h}^{-}\left(u_{m}\right), C_{3}$ and $C_{4}$ can be expressed in terms of $u_{m}$ and $u_{M}$ as

$q_{h}^{+}\left(u_{M}\right)=\frac{1}{a}\left(-b-C_{3} \mathrm{e}^{-a u_{M}}\right)=\frac{1}{a}\left(b-C_{4} \mathrm{e}^{a u_{M}}\right)=q_{h}^{-}\left(u_{M}\right)$ $q_{h}^{+}\left(u_{m}\right)=\frac{1}{a}\left(-b-C_{3} \mathrm{e}^{-a u_{m}}\right)=\frac{1}{a}\left(b-C_{4} \mathrm{e}^{a u_{m}}\right)=q_{h}^{-}\left(u_{m}\right)$ which yields

$$
\begin{aligned}
& C_{3}=b\left(\mathrm{e}^{a u_{m}}-\mathrm{e}^{a u_{M}}\right) \operatorname{csch} a\left(u_{M}-u_{m}\right) \\
& C_{4}=b\left(\mathrm{e}^{-a u_{m}}-\mathrm{e}^{-a u_{M}}\right) \operatorname{csch} a\left(u_{M}-u_{m}\right) .
\end{aligned}
$$

The work done can now be found to be

$$
W=2 \frac{b}{a^{2}}\left(a\left(u_{M}-u_{m}\right)-2 \tanh \frac{a}{2}\left(u_{M}-u_{m}\right)\right) .
$$

We note that the work is due to the term given in (12), and that the new formulation of the hysteresis model (15) yields the same work as found for the initial ColemanHogdon model (8) (Coleman and Hodgdon [1986]).

For the applied voltage signal above, the linearized sensitivity of (15) can be found from e.g.

$$
K_{q}=\frac{\left(c u_{M}+q_{h}^{+}\left(u_{M}\right)\right)-\left(c u_{m}+q_{h}^{+}\left(u_{m}\right)\right)}{u_{M}-u_{m}},
$$

using the expressions above. It is also notable that the parameter $\beta$ in (8) is the slope of $w$ at $u=0$ when $w_{0}=u_{0}=0$, thus $\beta$ and $K_{q}$ should be close in value.

\section{STATE OBSERVER}

In this section we develop a state observer for estimating the states of the system modeled in Section 2. The observer is used to to suppress noise and to cancel the bias present in the measurements. It will also mitigate the effect of the phase lag introduced by reconstruction and anti-aliasing filters. Utilizing state estimates should therefore improve the performance of the controller that is proposed in Section 4.

In the implementation we require knowledge of both displacement and velocity. However, a velocity measurement is unavailable, as we consider a system having only a displacement and a current sensor. A measure for the charge is obtained by integrating the current measurement over time. We will be employing the adaptive Luenberger observer (Ioannou and Sun [1995]), which we will tune using the steady-state Kalman filter and a nominal model. This should give us close to optimal state estimates in the presence of Gaussian, zero-mean, uncorrelated, and white noise (Simon [2006]).

The model for the system is found from (15) and (6). In the actual implementation of the system, both the displacement and the current measurement have unwanted time-varying bias components, and we therefore augment the system with three additional states in order to estimate the bias present in the displacement and current measurements. We thus have

$$
\dot{x}=f(x, u)=A(t) x+B u_{2},
$$

where

$$
A=\left[\begin{array}{cccccc}
0 & 1 & 0 & 0 & 0 & 0 \\
-\bar{a}_{0} & -a_{1} & \bar{b}_{0} & 0 & 0 & 0 \\
0 & 0 & -a|\dot{u}| & 0 & 0 & 0 \\
0 & 0 & 0 & 0 & 0 & 0 \\
0 & 0 & 0 & 0 & 0 & 1 \\
0 & 0 & 0 & 0 & 0 & 0
\end{array}\right], \quad B=\left[\begin{array}{cc}
0 & 0 \\
\bar{b}_{0} c & 0 \\
0 & -b \\
0 & 0 \\
0 & 0 \\
0 & 0
\end{array}\right]
$$

and

where

$$
y=h(x)=C x+D u_{2},
$$

$$
C=\left[\begin{array}{llllll}
1 & 0 & 0 & 1 & 0 & 0 \\
0 & 0 & 1 & 0 & 1 & 0
\end{array}\right], \quad D=\left[\begin{array}{ll}
0 & 0 \\
c & 0
\end{array}\right],
$$

$x=\left[x_{1}, x_{2}, q_{h}, x_{1 b}, q_{b}, i_{b}\right]^{\mathrm{T}}$ and $u_{2}=[u, \dot{u}]^{\mathrm{T}}$. Here $x_{1 b}$ is the bias present in the displacement measurement and $i_{b}$ is the bias present in the current measurement. Since we obtain a measure for the total charge in the system by integrating the current measurement over time, we also add a state $q_{b}$ to compensate for the time integral of $i_{b}$.

As will be explained in Section 6, we will have parameter estimates of $\bar{b}_{0}, a, b$, and $c$, but $\bar{a}_{0}$ and $a_{1}$ are fixed. Given the process noise $w_{n} \sim(0, Q)$, measurement noise $v_{n} \sim(0, R)$, and a nominal state matrix $\check{A}$, we find the steady-state covariance $P_{\infty}$ from the continuous algebraic Riccati equation

$$
-P_{\infty} C^{\mathrm{T}} R^{-1} C P_{\infty}+\check{A} P_{\infty}+P_{\infty} \check{A}^{\mathrm{T}}+Q=0 .
$$

The measurement noise is described by the variance of the signals from the displacement and the charge measurements recorded when $u=0$. The Kalman gain is then given by

$$
K=P_{\infty} C^{\mathrm{T}} R^{-1} .
$$

To ensure bounded state estimates, we employ a slightly modified version of the adaptive Luenberger observer. Having initial state estimates $\hat{x}_{0}=E\left[x_{0}\right]$, we find the state estimates $\hat{x}$ using:

$$
\dot{\hat{x}}=\hat{A}(t) \hat{x}+\hat{B}(t) u+L(t)(y-\hat{y}), \quad \hat{y}=C \hat{x}+\hat{D}(t) u
$$

Equation (20) can be put on the form

$$
\begin{aligned}
\dot{\hat{x}}=(\hat{A}-L C) \hat{x}+(\hat{B}-L \hat{D}) u+L C x & \\
& =A^{*} \hat{x}+(\hat{B}-L \hat{D}) u+L C x
\end{aligned}
$$

where we ensure that $A^{*}$ is a stable, time-invariant matrix. This is done by choosing

$$
\hat{A}-L C=A^{*}=\check{A}-K C \Rightarrow L C=K C+\hat{A}-\check{A}
$$

Depending on the structure of $C$, only some elements of $\hat{A}$ can be allowed to be time-varying. For the system at hand we select

$$
L(t)=K+T(t)
$$

where $T$ is a $6 \times 2$ matrix:

$$
T(t)=\left[\begin{array}{cccccc}
0 & 0 & 0 & 0 & 0 & 0 \\
0 & \hat{\bar{b}}_{0}-\check{\bar{b}}_{0} & \hat{a}|\dot{u}|-\check{a}|\check{\dot{u}}| & 0 & 0 & 0
\end{array}\right]^{\mathrm{T}}
$$

Here $\hat{\bar{b}}_{0}$ and $\hat{a}$ are (time-varying) parameter estimates, $\check{\bar{b}}_{0}$ and $\check{a}$ are nominal (constant) values. The nominal value for the term $|\dot{u}|$ is chosen to be the root-mean-square-value of the time derivative of the control signal when using reference feed-forward, and is denoted $|\check{\dot{u}}|$.

Boundedness of (20) is now ensured. We can choose $u, \dot{u} \in$ $\mathcal{L}_{\infty}$. We know from the solution of (15) that $q \in \mathcal{L}_{\infty}$. From e.g. Rouths criterion, we can immediately see that the mechanical model (6) is stable, thus $x_{1}, x_{2} \in \mathcal{L}_{\infty}$. For the parameter estimate $\hat{\theta}$, we also have $\hat{\theta} \in \mathcal{L}_{\infty}$, as will 
be shown in Section 6 . Now, since $A^{*}$ is a stable, timeinvariant matrix, we have that $\hat{x} \in \mathcal{L}_{\infty}$ (Ioannou and Sun [1995]).

\section{CONTROL}

In this section we propose to use an ISS (input-to-state stability) controller to achieve tracking. This controller benefits from robustness against model parameter error and quick convergence due to non-linear damping terms in the control law, and it allows for a modular design, where parameter identification can be done separately from the controller (Krstic et al. [1995]).

Considering (6) and (15) we obtain the system

$$
\begin{aligned}
& \dot{x}_{1}=x_{2} \\
& \dot{x}_{2}=-\bar{a}_{0} x_{1}-a_{1} x_{2}+\bar{b}_{0} c u+\bar{b}_{0} q_{h} .
\end{aligned}
$$

If we assume no knowledge of the hysteresis model (15) for now and consider the response of the system to be linear, the best we can do is to use

in (21) and obtain

$$
c u+q_{h}=K_{q} u \Rightarrow c u \approx K_{q} u
$$

$$
\begin{aligned}
& \dot{x}_{1}=x_{2} \\
& \dot{x}_{2}=-\bar{a}_{0} x_{1}-a_{1} x_{2}+\beta_{0} u+\bar{b}_{0} q_{h},
\end{aligned}
$$

where the control gain $\beta_{0}=\bar{b}_{0} K_{q}$, and we consider $\Delta_{q_{h}}=$ $\bar{b}_{0} q_{h}$, due to hysteresis, a bounded disturbance input to the system, as has been done in Su et al. [2000] and Zhou et al. [2004]. From (13) and (14) we see that the hysteretic behavior is bounded by $\left\|q_{h}\right\|_{\infty}=\frac{b}{a}$.

\subsection{Model Reduction}

Considering the approximation in (17), we are assuming that we will not excite any resonances in the positioning stage. We also have a constraint in available bandwidth in the hardware used for implementing the control scheme. Applying an ISS controller to the model in (23) yields a very high bandwidth requirement due to the non-linear damping terms. We must therefore use a time step in the numerical integration scheme which is much smaller than what is implementable. By not exciting any resonances we note that removing the high frequency dynamics from (23) still renders a very good approximation to the observed dynamics, and the resulting model will reduce the bandwidth requirement for the ISS controller.

If we find the Laplace transform of (23) as

$$
G(s)=\frac{\beta_{0}}{s^{2}+a_{1} s+\bar{a}_{0}},
$$

we approximate the low frequency dynamics $G_{l f}$ as

$$
G_{l f}=\frac{\beta}{s+\bar{a}_{0}^{\prime}}
$$

with $\bar{a}_{0}^{\prime}>0$, and then factor out the high frequency dynamics $G_{h f}$, such that

$$
G(s)=G_{l f}(s)+G_{h f}(s)
$$

and

$$
\lim _{s \rightarrow 0} s \frac{1}{s} G_{l f}=\lim _{s \rightarrow 0} s \frac{1}{s} G_{h f} \Rightarrow \frac{\beta_{0}}{\bar{a}_{0}}=\frac{\beta}{\bar{a}_{0}^{\prime}} .
$$

$G_{h f}$ is found as

$$
G_{h f}=\frac{\left(s-\bar{a}_{0}^{\prime}\right) \beta_{0}-\beta\left(s^{2}+a_{1} s+\bar{a}_{0}\right)}{\left(s+\bar{a}_{0}^{\prime}\right)\left(s^{2}+a_{1} s+\bar{a}_{0}\right)}
$$

which is a proper and stable transfer function, with a high pass characteristic,

$$
\lim _{s \rightarrow 0} s \frac{1}{s} G_{h f}=\frac{a_{0}^{\prime} \beta_{0}-\beta a_{0}}{a_{0}^{\prime} a_{0}}=\frac{\beta_{0}}{a_{0}}-\frac{\beta}{a_{0}^{\prime}}=0 .
$$

Choosing i.e. $\bar{a}_{0}^{\prime}=\sqrt{\overline{a_{0}}}$, yields $\beta=\frac{\beta_{0}}{\bar{a}_{0}} \bar{a}_{0}^{\prime}=\frac{\beta_{0}}{\bar{a}_{0}} \sqrt{\bar{a}_{0}}=\frac{\beta_{0}}{\sqrt{\bar{a}_{0}}}$.

The high frequency dynamics (24) can be seen as yet another bounded disturbance at the input of the system, $\Delta_{h f} \in \mathcal{L}_{\infty}$.

\subsection{ISS Controller}

Having the model

$$
\dot{x}_{1}=-\bar{a}_{0}^{\prime} x_{1}+\beta u+\Delta_{q_{h}}+\Delta_{h f}=\theta w_{1}+\beta u+\Delta
$$

we follow the development in Krstic et al. [1995] to find the ISS controller. We start by defining the error variable

$$
z_{1}=x_{1}-y_{r}
$$

where $y_{r}$ is the reference output we wish to track, and which derivative, $\dot{y}_{r}$ is known, bounded and piecewise continuous.

Given some estimate of the parameter $\hat{\theta}=\theta-\tilde{\theta}$ and the control gain $\hat{\beta}=\beta-\tilde{\beta}$ (where $\tilde{\theta}$ and $\tilde{\beta}$ denotes the respective errors), and the regressor vectors $w_{1}$, we find the stabilizing function $\alpha_{1}$, and non-linear damping term $s_{1}$ as

$$
\begin{aligned}
\bar{\alpha}_{1} & =-w_{1} \hat{\theta} \\
s_{1} & =\kappa_{1}\left[\left|w_{1}\right|^{2}+\left(\frac{\bar{\alpha}_{1}+\dot{y}_{r}}{\hat{\beta}}\right)^{2}\right] \\
\alpha_{1} & =\bar{\alpha}_{1}-\hat{\beta} \frac{\operatorname{sgn}(\beta)}{\varsigma}\left(c_{1}+s_{1}\right) z_{1}
\end{aligned}
$$

where we assume that $|\beta| \geq \varsigma$. Both the sign of $\beta$ and a lower bound, $\varsigma>0$, for $|\beta|$ must be known. The constants $c_{1}$ and $\kappa_{1}$ are the controller gains, and are used for tuning the controller.

The control law can now be stated as

$$
u=\frac{1}{\hat{\beta}}\left(\alpha_{1}+\dot{y}_{r}\right)
$$

which yields the error dynamics

$$
\begin{aligned}
\dot{z}_{1}= & \dot{x}_{1}-\dot{y}_{r}=w_{1} \theta+\beta u-\dot{y}_{r}+\Delta \\
= & w_{1} \theta+\frac{\beta}{\hat{\beta}}\left(\alpha_{1}+\dot{y}_{r}\right)-\dot{y}_{r}+\Delta \\
= & w_{1} \theta+\frac{\beta}{\hat{\beta}}\left(\bar{\alpha}_{1}+\dot{y}_{r}\right)-\frac{|\beta|}{\varsigma}\left(c_{1}+s_{1}\right) z_{1}-\dot{y}_{r}+\Delta \\
= & w_{1} \theta+\bar{\alpha}_{1}+\frac{\tilde{\beta}}{\hat{\beta}}\left(\bar{\alpha}_{1}+\dot{y}_{r}\right)-\frac{|\beta|}{\varsigma}\left(c_{1}+s_{1}\right) z_{1}+\Delta \\
= & w_{1} \tilde{\theta}+\frac{\tilde{\beta}}{\hat{\beta}}\left(\bar{\alpha}_{1}+\dot{y}_{r}\right)-\frac{|\beta|}{\varsigma}\left(c_{1}+s_{1}\right) z_{1}+\Delta \\
= & w_{1} \tilde{\theta}-\frac{|\beta|}{\varsigma} \kappa_{1}\left|w_{1}\right|^{2} z_{1} \\
& +\frac{\tilde{\beta}}{\hat{\beta}}\left(\bar{\alpha}_{1}+\dot{y}_{r}\right)-\frac{|\beta|}{\varsigma} \kappa_{1}\left(\frac{\bar{\alpha}_{1}+\dot{y}_{r}}{\hat{\beta}}\right)^{2} z_{1}-\frac{|\beta|}{\varsigma} c_{1} z_{1}+\Delta .
\end{aligned}
$$

If we consider the Lyapunov function $V=\frac{1}{2} z_{1}^{2}$, we find using (26) that the total derivative of $V$ is 


$$
\begin{aligned}
\dot{V}= & z_{1} \dot{z}_{1} \\
= & w_{1} \tilde{\theta} z_{1}-\frac{|\beta|}{\varsigma} \kappa_{1}\left|w_{1}\right|^{2} z_{1}{ }^{2}+\frac{\tilde{\beta}}{\hat{\beta}}\left(\bar{\alpha}_{1}+\dot{y}_{r}\right) z_{1} \\
& -\frac{|\beta|}{\varsigma} \kappa_{1}\left(\frac{\bar{\alpha}_{1}+\dot{y}_{r}}{\hat{\beta}}\right)^{2} z_{1}{ }^{2}-\frac{|\beta|}{\varsigma} c_{1} z_{1}{ }^{2}+\Delta z_{1} .
\end{aligned}
$$

By using Young's equality, the following upper bound on $\dot{V}$ can be found

$$
\dot{V} \leq-\frac{|\beta|}{2 \varsigma} c_{1} z_{1}^{2}+\frac{\varsigma|\tilde{\beta}|^{2}}{4 \kappa_{1}|\beta|}+\frac{\varsigma|\tilde{\theta}|^{2}}{4 \kappa_{1}|\beta|}+\frac{\varsigma|\Delta|^{2}}{2 c_{1}|\beta|},
$$

which means that $z=z_{1}$ is globally uniformly bounded, if $\tilde{\theta}, \tilde{\beta}, \Delta \in \mathcal{L}_{\infty}$. If the parameter errors, $\tilde{\theta}=0, \tilde{\beta}=0$, and the disturbance, $\Delta=0$, then $z_{1}=0$ is globally asymptotically stable (Krstic et al. [1995]). The error, z, is bounded by

$$
|z| \leq\left|z_{0}\right| \mathrm{e}^{-\frac{|\beta|}{\varsigma} c_{1} t}+\frac{1}{\sqrt{c_{1}}}\left(\frac{\|\tilde{\theta}\|_{\infty}^{2}}{2 \kappa_{1}}+\frac{\|\tilde{\beta}\|_{\infty}^{2}}{2 \kappa_{1}}+\frac{\|\Delta\|_{\infty}^{2}}{c_{1}}\right)^{\frac{1}{2}} .
$$

Reducing the parameter errors and the disturbance, or by using larger gains for $c_{1}$ and $\kappa_{1}$, tracking performance can be increased. Increasing the gain, on the other hand, will amplify noise present in measurements or estimates of $x$ and increase the adverse effects of systematic measurement and estimation errors.

When using the hysteresis compensation scheme presented in the next section, we require knowledge of the time derivative of the control signal. An approximation to $\dot{u}$, assuming $\dot{\hat{\beta}} \approx 0$, can be found as:

$$
\begin{aligned}
\dot{w}_{1} & =\dot{x}_{1}, \quad \dot{\bar{\alpha}}_{1}=-\dot{w}_{1} \hat{\theta} \\
\dot{\alpha}_{1} & =\dot{\bar{\alpha}}_{1}-\hat{\beta} \frac{\operatorname{sgn}(\beta)}{\varsigma}\left(\left(c_{1}+s_{1}\right) \dot{z}_{1}+\dot{s}_{1} z_{1}\right) \\
\dot{s}_{1} & =\kappa_{1}\left[2 \dot{w}_{1} w_{1}+2\left(\frac{\bar{\alpha}_{1}+\dot{y}_{r}}{\hat{\beta}}\right)\left(\frac{\dot{\bar{\alpha}}_{1}+\ddot{y}_{r}}{\hat{\beta}}\right)\right] \\
\dot{u} & =\frac{1}{\hat{\beta}}\left(\dot{\alpha}_{1}+\ddot{y}_{r}\right)
\end{aligned}
$$

\section{HYSTERESIS COMPENSATION}

Next, we propose a method for compensating for the hysteretic behavior of the actuator in a feed-forward fashion. By using

$$
u^{*}=\frac{K_{q}}{c} u-\frac{1}{c} \hat{q}_{h},
$$

where $\hat{q}_{h}$ is a estimate of $q_{h}$, as the new control law, and by substituting (27) in (21), we obtain

$$
\begin{aligned}
& \dot{x}_{1}=x_{2} \\
& \dot{x}_{2}=-\bar{a}_{0} x_{1}-a_{1} x_{2}+\bar{b}_{0} K_{q} u-\bar{b}_{0} \hat{q}_{h}+\bar{b}_{0} q_{h},
\end{aligned}
$$

where the last three terms of (28) can be written as

$$
\beta_{0} u+\bar{b}_{0}\left(q_{h}-\hat{q}_{h}\right)=\beta_{0} u+\tilde{\Delta}_{q_{h}},
$$

indicating that the disturbance $\Delta_{q_{h}}=\bar{b}_{0} q_{h}$ in (23) can be reduced to $\tilde{\Delta}_{q_{h}}<\Delta_{q_{h}}$, given a good estimate $\hat{q}_{h}$.

An open-loop observer to estimate $q_{h}$ can be obtained from (15), where we now use $\dot{u}^{*}$ as the input. The estimate $\hat{q}_{h}$ is then found as

$$
\begin{aligned}
\dot{\hat{q}}_{h}=-b \dot{u}^{*}- & a \hat{q}_{h}\left|\dot{u}^{*}\right| \\
& =-b\left(\frac{K_{q}}{c} \dot{u}-\frac{1}{c} \dot{\hat{q}}_{h}\right)-a \hat{q}_{h}\left|\frac{K_{q}}{c} \dot{u}-\frac{1}{c} \dot{\hat{q}}_{h}\right|
\end{aligned}
$$

which we can write as:

$$
\dot{\hat{q}}_{h}=\left\{\begin{array}{l}
K_{q} \frac{-a \hat{q}_{h}-b}{-a \hat{q}_{h}-b+c} \dot{u}, \dot{u}^{*} \geq 0 \\
K_{q} \frac{a \hat{q}_{h}-b}{a \hat{q}_{h}-b+c} \dot{u}, \dot{u}^{*}<0
\end{array}\right.
$$

Equation (29) can again be solved explicitly by separation of variables by cancelling the dependence on time. For the case $\dot{u}^{*} \geq 0$, we have

$$
\frac{\mathrm{d} \hat{q}_{h}}{\mathrm{~d} u}=K_{q} \frac{-a \hat{q}_{h}-b}{-a \hat{q}_{h}-b+c}
$$

which solution is found as:

$$
\begin{gathered}
\hat{q}_{h}-\frac{c}{a} \ln \left(a \hat{q}_{h}+b\right)=K_{q} u+C_{5} \\
C_{5}=\hat{q}_{h 0}-\frac{c}{a} \ln \left(a \hat{q}_{h 0}+b\right)-K_{q} u_{0}
\end{gathered}
$$

This implicit equation can be solved explicitly for $\hat{q}_{h}$ by using the Lambert $W$ function (Corless et al. [1996]), denoted $W(\cdot)$ :

$$
\hat{q}_{h}=-\frac{c}{a} W\left(-\frac{1}{c} \mathrm{e}^{-\left(\frac{a K_{q} u}{c}+\frac{a C_{1}}{c}+\frac{b}{c}\right)}\right)-\frac{b}{a}
$$

Similarly, for the case $\dot{u}^{*}<0$ we have

$$
\begin{gathered}
\hat{q}_{h}+\frac{c}{a} \ln \left(-a \hat{q}_{h}+b\right)=K_{q} u+C_{6}, \\
C_{6}=\hat{q}_{h 0}+\frac{c}{a} \ln \left(-a \hat{q}_{h 0}+b\right)-K_{q} u_{0},
\end{gathered}
$$

and the explicit solution is found as

$$
\hat{q}_{h}=\frac{c}{a} W\left(-\frac{1}{c} \mathrm{e}^{\left(\frac{a K_{q} u}{c}+\frac{a C_{2}}{c}-\frac{b}{c}\right)}\right)+\frac{b}{a} .
$$

Proposition 1. The solution of (29), $\hat{q}_{h}$, is bounded.

Proof. Differentiating (30) or (31) by $u$ yields $\frac{\mathrm{d} \hat{q}_{h}}{\mathrm{~d} u}<0$, thus $\dot{u} \geq 0 \Rightarrow \dot{\hat{q}}_{h} \leq 0$, and $\dot{u} \leq 0 \Rightarrow \dot{\hat{q}}_{h} \geq 0$. Therefore $\dot{u}^{*} \geq 0 \Rightarrow \dot{u} \geq 0$ and $\dot{u}^{*}<0 \Rightarrow \dot{u}<0$. Thus $\left\|\hat{q}_{h}\right\|_{\infty}=\frac{b}{a}$.

\section{PARAMETER IDENTIFICATION}

\subsection{Model Parameters from Frequency Response Data}

The parameters for the models (4), (5), and (6), were initially identified using frequency response data and the System Identification Toolbox in MATLAB. The frequency response data was collected using a SRS SR780 Dynamic Signal Analyzer. The identified parameters are presented in Table 1.

Table 1. Identified parameters for the models (4), (5), and (6).

\begin{tabular}{|c|c|c|}
\hline Parameter & Value & Unit \\
\hline$b_{0}$ & 2.07 & $\mathrm{~m} /\left(\mathrm{s}^{2} \mathrm{~V}\right)$ \\
\hline $\bar{b}_{0}$ & $6.24 \times 10^{6}$ & $\mathrm{~m} /\left(\mathrm{s}^{2} \mathrm{C}\right)$ \\
\hline$a_{0}$ & $1.08 \times 10^{8}$ & $1 / \mathrm{s}^{2}$ \\
\hline $\bar{a}_{0}$ & $1.17 \times 10^{8}$ & $1 / \mathrm{s}^{2}$ \\
\hline$a_{1}$ & 491 & $1 / \mathrm{s}$ \\
\hline$C_{a}$ & $332 \times 10^{-9}$ & $\mathrm{~F}$ \\
\hline$K_{a}$ & 1.36 & $\mathrm{~N} / \mathrm{V}$ \\
\hline
\end{tabular}

\subsection{Online Parameter Identification}

In order to increase tracking performance, we identify the model parameters online using the modified leastsquares adaptive law with forgetting factor, as described in Ioannou and Sun [1995]. This method provides good 
Table 2. Modified least-squares adaptive law with forgetting factor.

\begin{tabular}{|l|l|}
\hline Parametric model & $z=\theta^{\mathrm{T}} \Phi$ \\
\hline Estimation model & $\hat{z}=\hat{\theta}^{\mathrm{T}} \Phi$ \\
\hline Estimation error & $\epsilon=\frac{z-\hat{z}}{m^{2}}$ \\
\hline Adaptive law & $\dot{\hat{\theta}}=P \epsilon \Phi$ \\
& $\dot{P}= \begin{cases}\lambda P-\frac{P \Phi \Phi^{\mathrm{T}} P}{m^{2}}, & \text { if }\|P\| \leq R_{0} \\
0 & \text { otherwise } \\
& P(0)=P_{0}\end{cases}$ \\
\hline
\end{tabular}

parameter estimates in the presence of sensor noise. The method is summarized in Table 2.

Here $z$ is the output of the parametric model, $\theta$ is the parameter vector, and $\Phi$ is the regressor vector. The output of the parametric model given the vector of estimated parameters, $\hat{\theta}$, is denoted $\hat{z}$. $P$ is the covariance matrix, and the initial covariance matrix must be symmetric and positive definite, $P(0)=P_{0}=P_{0}^{\mathrm{T}}>0$. To avoid that $P$ becomes arbitrarily small in some directions in the space spanned by the parameter estimates, thus making the adaptation slow, the forgetting factor $\lambda$ is used, but this can cause $P$ to grow without bound, thus $\|P\|$ is bounded by $R_{0}$. The initial covariance matrix should therefore also satisfy $\|P(0)\| \leq R_{0}$. Normalization is achieved using the signal $m^{2}$, which can be taken to be

$$
m^{2}=1+n_{s}{ }^{2}, n_{s}{ }^{2}=\Phi^{\mathrm{T}} P \Phi .
$$

The normalized least-squares algorithm have the properties $\epsilon, \epsilon n_{s}, \hat{\theta}, \dot{\hat{\theta}}, P \in \mathcal{L}_{\infty}$. Thus the parameter estimate error $\tilde{\theta}$ is bounded, which is required by the ISS controller.

\subsection{Hysteresis Model Parameters}

Setting $w=q$ in the hysteresis model in (8), we have

$$
\dot{q}=\beta \dot{u}-\alpha q|\dot{u}|+\gamma|\dot{u}| u,
$$

and we see that by choosing

$$
\begin{aligned}
\theta & =(\alpha, \beta, \gamma)^{\mathrm{T}} \\
\Phi & =(-w|\dot{u}|, \dot{u},|\dot{u}| u)^{\mathrm{T}}
\end{aligned}
$$

the model can be put in the parametric form $z=\theta^{\mathrm{T}} \Phi$, where $z=\dot{q}$. Having a measurement of the total current flowing in the piezoelectric actuator, $z$ is a known signal. The charge $q$ can then be found by integrating $\dot{q}$ over time, and since the control input $u$ and its derivative $\dot{u}$ is known, the signals in $\Phi$ can be generated.

Identified parameters for the hysteresis model are given in Table 3. Given the parameter estimates $\hat{\alpha}, \hat{\beta}$, and $\hat{\gamma}$, the parameter estimates $\hat{a}, \hat{b}$, and $\hat{c}$ for $(15)$, are found from (16). Figure 3 presents the measured response compared to the computed response of the model (15). As can be seen, the model with the identified parameters provide a very good fit to the actual behavior.

Table 3. Identified parameters for (8).

\begin{tabular}{|c|c|}
\hline Parameter & Value \\
\hline$\alpha$ & $3.96 \times 10^{-2}$ \\
\hline$\beta$ & $4.32 \times 10^{-1}$ \\
\hline$\gamma$ & $2.06 \times 10^{-2}$ \\
\hline
\end{tabular}

\subsection{Other Model Parameters}

In the implementation of the control law (25), identifying the parameters $\bar{a}_{0}^{\prime}$ and $\beta$ should provide better tracking.

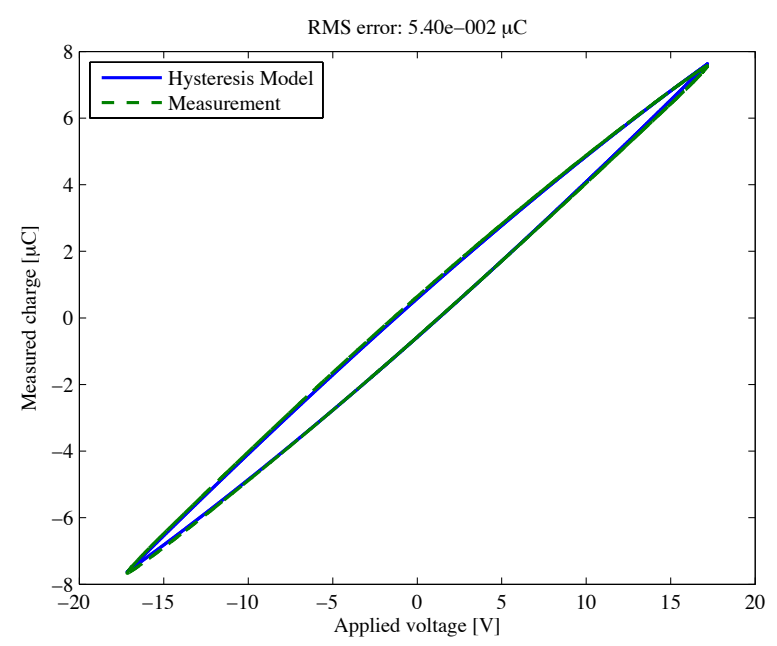

Fig. 3. Experiment: Applied voltage vs. measured charge and applied voltage vs. model with identified parameters.

For the hysteresis compensation, given by (27) and (29), we find the parameters $a, b$, and $c$ from the scheme in Section 6.3 , but in addition we also need an estimate of the parameter $K_{q}$. For the state observer, we require estimates the parameters $\bar{b}_{0}, \bar{a}_{0}, a_{1}, a, b$, and $c$.

We expect the mass, damping, and stiffness of the system to be constant, and since we are exiting the system at frequencies well below the first resonance frequency, we decided to fix the parameters $\bar{a}_{0}$ and $a_{1}$, in order to reduce the complexity of the parameter identification scheme.

To obtain an estimate of $K_{q}$, we could use (19), but this requires knowledge of $u_{M}$ and $u_{m}$, for which there is no obvious solution for an arbitrary input signal $u$. We therefore estimate $K_{q}$ by using

$$
q=K_{q} u \text {. }
$$

With $z=q$, the equation is put on parametric form by choosing

$$
\begin{aligned}
\theta & =K_{q} \\
\Phi & =u .
\end{aligned}
$$

To obtain estimates of $\beta$ and $\bar{b}_{0}$, we note that $\beta=\frac{\bar{b}_{0} K_{q}}{\sqrt{\bar{a}_{0}}}$, given that $\bar{a}_{0}^{\prime}=\sqrt{\bar{a}_{0}}$, which is fixed. Thus we only need to estimate $\bar{b}_{0}$.

Putting (6) on the form

$$
z=x_{1}=\frac{\bar{b}_{0}}{s^{2}+a_{1} s+\bar{a}_{0}} q=\bar{b}_{0} W(s) q,
$$

we find the parametric form by choosing

$$
\begin{aligned}
\theta & =\bar{b}_{0} \\
\Phi & =W(s) q .
\end{aligned}
$$

\section{EXPERIMENTS}

The performance of the hysteresis compensation, given by (27) and (29), and the backstepping control law (25) using state estimates from the state observer (20), was assessed by experiments. 


\subsection{Description of the Experimental System}

The nanopositioning device utilized is a serial kinematic device with one moving platform suspended by leaf flexures and actuated by a $10 \mathrm{~mm}$ Noliac SCMAP07 stack actuator. It is a simplified design based on the device described in Leang and Fleming [2009]. Displacement was measured using a SIOS Meßtechnik SP120 interferometer $(0.24 \mu \mathrm{m} / \mathrm{V})$. The actuator was driven using a Piezodrive PDL200 linear voltage amplifier $(20 \mathrm{~V} / \mathrm{V})$. Current was measured using an inverting op-amp circuit with a BurrBrown OPA2111 and a $10 \mathrm{k} \Omega$ resistor, thus having a sensitivity of $-0.1 \mu \mathrm{A} / \mathrm{V}$. Everything was implemented on a dSPACE DS1103 harware-in-the-loop board.

\subsection{Results}

The first experiment is presented in Fig. 4. The reference is just feed forwarded, using

$$
u=\frac{1}{\beta_{0}} y_{r} \text { and } \dot{u}=\frac{1}{\beta_{0}} \dot{y}_{r},
$$

where $\beta_{0}=\bar{b}_{0} K_{q}$. This provides an upper bound for the error. The second experiment is presented in Fig. 5 and 6 , where we have reference feed forward and hysteresis compensation. As can be seen, the hysteresis compensation cancels the effect of the hysteresis well, and the root-meansquare (RMS) error is reduced by more than an order of magnitude. Next, the ISS controller is used. The gains of the controller were set to $c_{1}=20000$ and $\kappa_{1}=10$. The resulting time-series is presented in Fig. 7. The ISS controller with these gains provides better tracking, but some hysteresis is still left. Lastly, the ISS controller was used in conjunction with the hysteresis compensation scheme, and the result is presented in Fig. 8. The error is seen to be marginally worse than when using feed forward and hysteresis compensation. Experience with the implemented system showed that the ISS controller with hysteresis compensation and feed forward with hysteresis compensation yielded about the same error over time when the parameter estimates had converged. In general the scheme using the ISS controller yielded a smaller error when some parameter estimation error was present.

The RMS error obtained from the different experiments are summarized in Table 4.

Table 4. RMS error summary.

\begin{tabular}{|rcc|}
\hline Feed forward: & $2.43 \times 10^{-2}$ & $\mu \mathrm{m}$ \\
\hline Feed forward and hysteresis compensation: & $1.53 \times 10^{-3}$ & $\mu \mathrm{m}$ \\
\hline ISS controller: & $5.49 \times 10^{-3}$ & $\mu \mathrm{m}$ \\
\hline ISS controller and hysteresis compensation: & $1.55 \times 10^{-3}$ & $\mu \mathrm{m}$ \\
\hline
\end{tabular}

\section{CONCLUSIONS}

The implementation of the proposed hysteresis compensation scheme presented in this paper has been shown through experiment to reduce the hysteretic behavior of a piezoelectric actuator significantly. The scheme is simple to implement, and since the parameters used in the scheme are identified online, the method will provide good performance even if the parameters change, e.g. due to depolarization of material, or aging.

The proposed adaptive backstepping controller using estimated states, also in conjunction with the hysteresis compensation scheme, was shown through experiments to be a feasible solution for providing tracking control. Using the

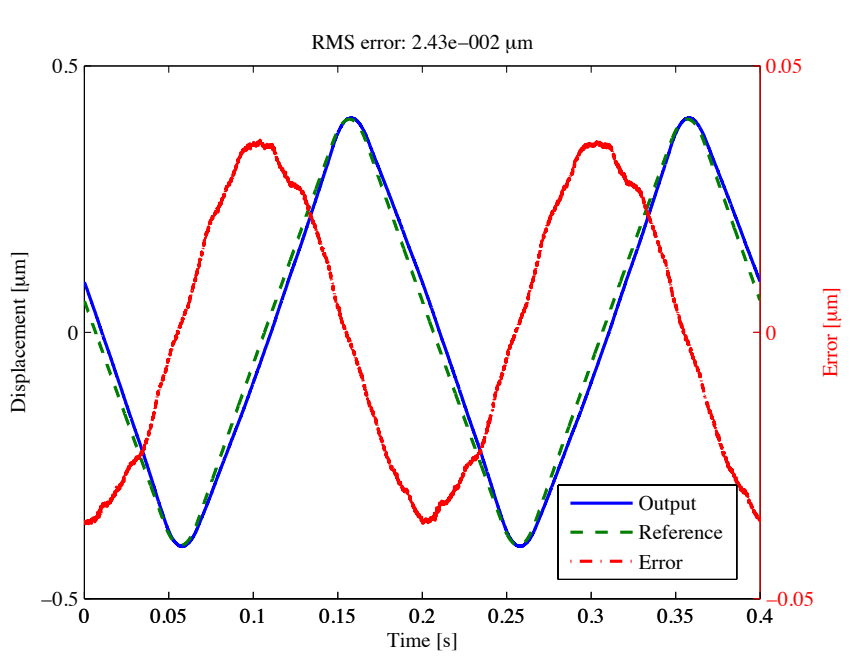

Fig. 4. Experiment: Feed forward.

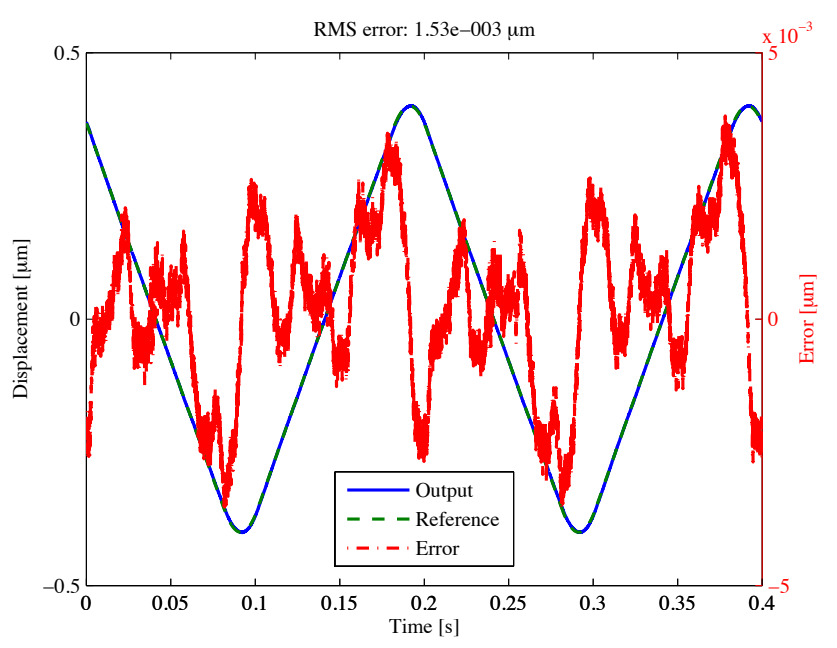

Fig. 5. Experiment: Feed forward and hysteresis compensation.

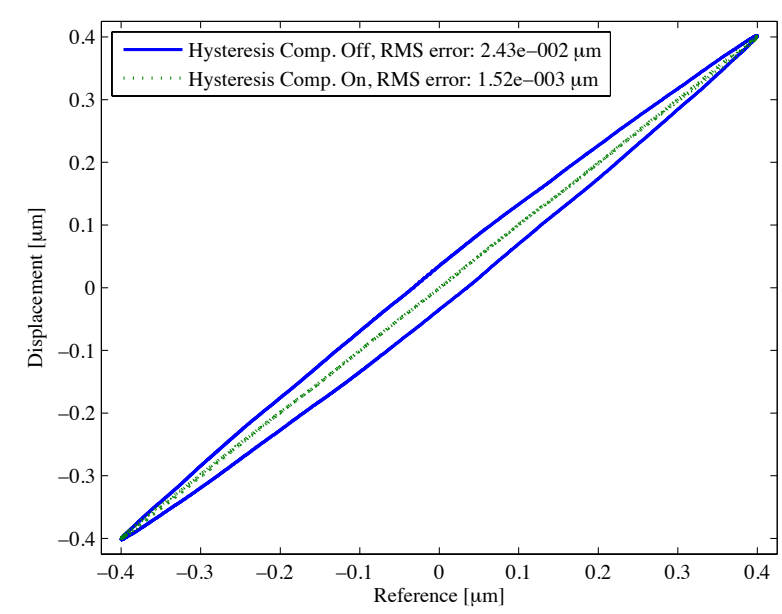

Fig. 6. Experiment: Reference vs. output, without and with hysteresis compensation. 


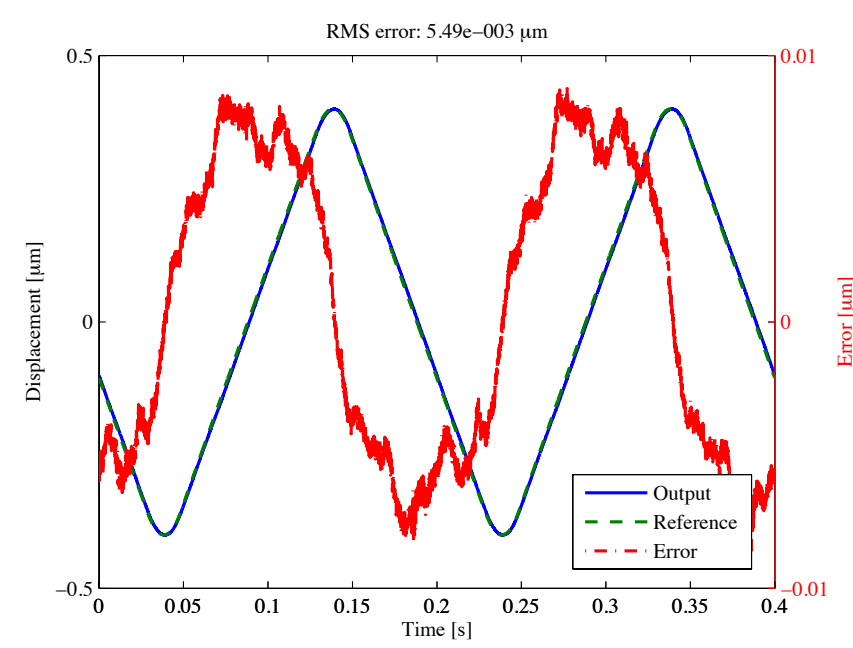

Fig. 7. Experiment: ISS controller.

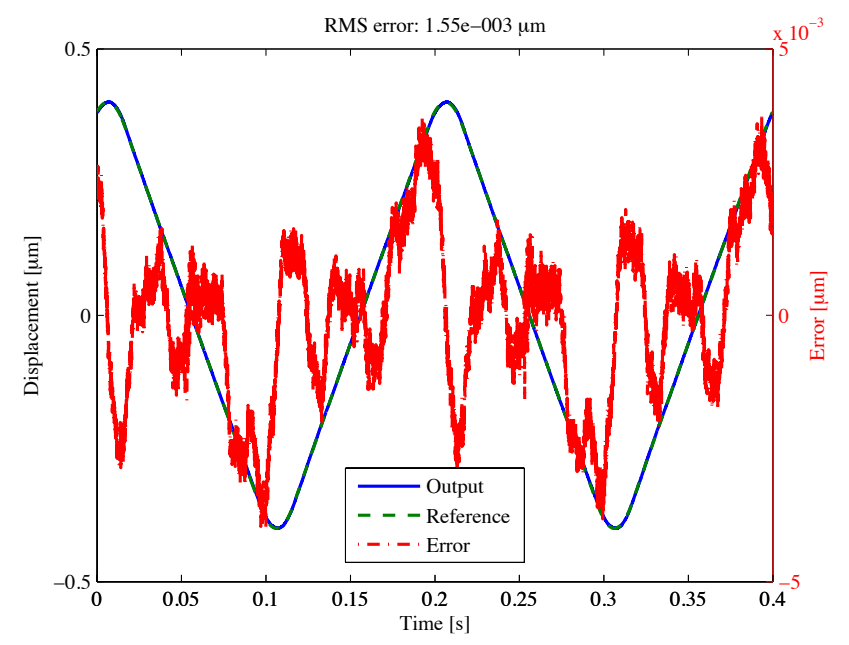

Fig. 8. Experiment: ISS controller and hysteresis compensation.

controller with estimated states is beneficial when noise is present in the measurements. The presence of anti-aliasing and reconstruction filters degrade performance, but using the state observer mitigates this effect. Being an adaptive controller, changes in the parameters of the system can be accounted for.

Using the backstepping controller in conjunction with the hysteresis compensation scheme when parameter estimates had converged provided no additional reduction in error over the case when using reference feed forward and hysteresis compensation. The controller improved performance when some parameter estimation error was present.

\section{FURTHER WORK}

During work on the actual implementation, some issues has come to light that should be given more consideration.

When using controller with state estimates where bias components are removed, absolute positioning with respect to an initial position reference is not possible. That is, low frequency drift due to other factors than drift introduced by the employed measurement technique is not observable.
The hysteresis compensation scheme requires knowledge of the time derivative of the control signal. When using the ISS controller, generating this signal without resorting to numeric differentiation requires a good estimate of the velocity of the system. Having poor parameter estimates, e.g. by introducing an error of $1 \%$ in the parameter estimates, typically lead to poor velocity estimation, which again produced a poor reproduction of the time derivative of the control signal. This degraded the robustness of the control scheme, which resulted in poor tracking performance.

It should also be mentioned that parameter identification with projection should be used to make absolutely sure that the bounds for the parameters in the hysteresis model (8) and the control gain estimate $\hat{\beta}$ in the control law (25) are not violated.

\section{REFERENCES}

Banning, R., de Koning, W., Adriaens, H., and Koops, R. (2001). State-space analysis and identification for a class of hysteretic systems. Automatica, 37(12), 1883-1892.

Coleman, B. and Hodgdon, M. (1986). A constitutive relation for rate-independent hysteresis in ferromagnetically soft materials. Int J Eng Sci, 24(6), 897-919.

Corless, R., Gonnet, G., Hare, D., Jeffrey, D., and Knuth, D.E. (1996). On the LambertW function. Advances in Computational Mathematics, 5(1), 329-359. doi: 10.1007/BF02124750.

Dahl, P.R. (1968). A solid friction model. Technical Report TOR-0158(310\%-18)-1, 1-31.

Devasia, S., Eleftheriou, E., and Moheimani, S.O.R. (2007). A survey of control issues in nanopositioning. Control Systems Technology, 15(5), 802-823.

Ioannou, P.A. and Sun, J. (1995). Robust Adaptive Control. Prentice Hall, Inc.

Krstic, M., Kanellakopoulos, I., and Kokotovic, P.V. (1995). Nonlinear and Adaptive Control Design. WileyInterscience.

Leang, K.K. and Fleming, A.J. (2009). High-speed serialkinematic spm scanner: design and drive considerations. Asian journal of control, 11(2), 144-153. doi: 10.1002/asjc.90.

Newcomb, C. and Flinn, I. (1982). Improving the linearity of piezoelectric ceramic actuators. Electronics Letters, 18(11), 442-443.

Preumont, A. (2006). Mechatronics. Springer.

Simon, D. (2006). Optimal State Estimation. WileyInterscience.

Stepanenko, Y. and Su, C.Y. (1998). Intelligent control of piezoelectric actuators. IEEE Conference on Decision and Control, 37th, 4234-4239.

Su, C., Stepanenko, Y., Svoboda, J., and Leung, T. (2000). Robust adaptive control of a class of nonlinear systems with unknown backlash-like hysteresis. Automatic Control, IEEE Transactions on, 45(12), 2427 - 2432. doi: $10.1109 / 9.895588$.

Zhou, J., Wen, C., and Zhang, Y. (2004). Adaptive backstepping control of a class of uncertain nonlinear systems with unknown backlash-like hysteresis. Automatic Control, IEEE Transactions on, 49(10), 1751- 1759. 